\title{
PHONON CONFINEMENT AND ELECTRON CAPTURE TIME IN QUANTUM WELL
}

\author{
K. KÁLNA \\ Institute of Electrical Engineering, Slovak Academy of Sciences \\ Dúbravská cesta 9,842 39 Bratislava, Slovakia
}

\begin{abstract}
The electron capture time via an electron-polar optical phonon interaction is calculated considering the confinement of a phonon in a GaAs quantum well laser structure. The effect of the phonon confinement decreases the electron capture time about twice comparing the electron capture time obtained from the interaction of an electron with the bulk phonon.
\end{abstract}

PACS numbers: 73.20.Dx, 73.61.Ey, 72.15.Nj

\section{Introduction}

Carrier capture times determine the rebuilding of an inversion population in a confinement region of semiconductor quantum well (QW) lasers and play a key role in their efficiency. Previous quantum-mechanical calculations [1-3] have revealed that the electron capture time oscillates as a function of QW width and that the most important scattering mechanism is due to electron-polar optical phonon (e-pop) interaction. So far, the polar optical phonon (pop) has been assumed to be a bulk phonon. In oscillation minima, the electron-electron (e-e) interaction-assisted capture time can improve the electron capture efficiency [3]. The electron capture constitutes a limiting factor in the carrier capture process into the $\mathrm{QW}$ because holes are captured in the QW first due to their larger effective mass $[3,4]$.

For the QW width smaller than $100 \AA$ [5], a confinement of phonons should be taken into account together with the electron confinement. We incorporate a model proposed by Ridley [6] into the previous calculations of the e-pop capture time $[2,3]$. Ridley's model has introduced a new quasi-particle, the hybridon, to collect three types of optical phonon modes; longitudinal, transversal and interface; in the semiconductor QW. An electron-hybridon (e-hy) interaction is considered only in the QW region, whereas over the AlGaAs barrier we still take into consideration the interaction of an electron with the bulk pop owing to the large barrier width [5]. We will abbreviate this mixed capture time as the e-hy+pop capture rate.

In Ref. [5], for the GaAs $/ \mathrm{Ga}_{x} \mathrm{In}_{1-x}$ As QW structure with $x=0.3$ and the $\mathrm{QW}$ width equal to $50 \AA$, the e-hy+pop interaction-induced capture rate from the 
lowest energy level in the barrier into the one in the QW was found to be twice slower than the electron capture rate via the interaction with bulk phonons only. In this paper, we argue that the entire e-hy+pop interaction-induced capture time is about twice shorter than the entire e-pop interaction-induced capture time even in the resonance minima. This fact improves the electron capture time which can be further improved via the electron-electron (e-e) interaction in the resonance minima.

\section{Electron-hybridon scattering rate}

We consider a separate confinement heterostructure quantum well (SCHQW) in our calculations. The SCHQW contains a GaAs QW of width $w$ between two $\mathrm{Al}_{x} \mathrm{Ga}_{1-x} \mathrm{As}$ barriers with a thickness $b=500 \AA$. From both sides there are thick AlAs cladding layers. Aluminium content $x$ is chosen so that it corresponds to $0.3 \mathrm{eV} \mathrm{QW} \mathrm{depth.} \mathrm{The} \mathrm{lattice} \mathrm{temperature} \mathrm{is} 8 \mathrm{~K}$ and the electron density in the $\mathrm{QW}$ is $N_{\mathrm{S}}=10^{15} \mathrm{~m}^{-2}$.

The e-hy interaction can be expressed using the Fermi golden rule. We can write the e-hy scattering rate of an electron with the wave vector $k$ from the subband $i$ to the subband $m$ for a spontaneous phonon emission only using the hybridization model of Ref. [6] as follows:

$$
\lambda_{i m}^{\mathrm{e}-\mathrm{hy}}(k)=\frac{e^{2} \omega_{\mathrm{LO}} m_{\mathrm{e}}}{2 \pi \hbar^{2} w}\left(\frac{1}{\kappa^{\mathrm{W}} \infty}-\frac{1}{\kappa^{\mathrm{W}}}\right) \int_{0}^{2 \pi} \mathrm{d} \theta \sum_{Q} \frac{\left[F_{i m(\text { parity) }}^{\mathrm{e}-\mathrm{hy}}(Q, q)\right]^{2}}{K_{\text {(parity) }}^{2}(Q, q)},
$$

In the above expression the symmetric (s) phonon mode takes place when $i+m$ is an even number and the antisymmetric (a) phonon mode when $i+m$ is an odd number, $m_{\mathrm{e}}$ is the electron effective mass, $\kappa^{\mathrm{W}}$ and $\kappa_{\infty}^{\mathrm{W}}$ are the respective static and high-frequency permittivities in the QW, and $\hbar \omega_{L O}$ is the energy of the longitudinal optical phonon in GaAs. The parameter $K_{(\mathrm{s})}(Q, q)$ for the symmetric phonon mode may be obtained from the equation

$$
\begin{gathered}
K_{(\mathrm{s})}^{2}(Q, q)=Q^{2}\left[1-\frac{\sin (Q w)}{Q w}\right]+q^{2}\left[1+\frac{\sin (Q w)}{Q w}\right] \\
-4 p_{2} \frac{q}{w}\left[1+\cos (Q w)-p_{2} \cos ^{2} \frac{Q w}{2} \operatorname{coth} \frac{q w}{2}\right]
\end{gathered}
$$

and the parameter $K_{(\mathrm{a})}(Q, q)$ for the antisymmetric phonon mode from the equation

$$
\begin{gathered}
K_{(\mathrm{a})}^{2}(Q, q)=Q^{2}\left[1+\frac{\sin (Q w)}{Q w}\right]+q^{2}\left[1-\frac{\sin (Q w)}{Q w}\right] \\
-4 p_{1} \frac{q}{w}\left[1-\cos (Q w)-p_{1} \sin ^{2} \frac{Q w}{2} \tanh \frac{q w}{2}\right] .
\end{gathered}
$$

In Eqs. (2) and (3) the following quantities are used:

$$
q=\left[2 k^{2}+\frac{2 m_{\mathrm{e}}}{\mathrm{h}^{2}} E_{\mathrm{S}}^{\mathrm{P}}-2 k\left(k^{2}+\frac{2 m_{\mathrm{e}}}{\mathrm{h}^{2}} E_{\mathrm{S}}^{\mathrm{P}}\right)^{1 / 2} \cos \theta\right]^{1 / 2},
$$




$$
\begin{aligned}
& p_{1}=\frac{1}{t[\tanh (q w / 2)+r]}, \quad p_{2}=\frac{1}{t[\operatorname{coth}(q w / 2)+r]}, \\
& r=\frac{\kappa_{\infty}^{\mathrm{W}}}{\kappa_{\infty}^{\mathrm{B}}} \frac{\omega^{2}-\omega_{\mathrm{LO}}^{2}}{\omega^{2}-\omega_{\text {TO }}^{2}}, \quad t=\frac{\omega^{2}-\omega_{\mathrm{LO}}^{2}}{\omega^{2}-\omega_{\mathrm{TO}}^{2}}, \quad \omega^{2}=\omega^{2}-v_{\mathrm{L}}{ }^{2}\left(q^{2}+Q^{2}\right),
\end{aligned}
$$

where $E_{\mathrm{S}}^{\mathrm{P}}=E_{i}-E_{m}-\hbar \omega_{\mathrm{LO}}, \hbar \omega_{\mathrm{T}}$ is the energy of the transversal optical phonon, $v_{\mathrm{L}}$ the sound velocity, and $\kappa_{\infty}^{\mathrm{B}}$ the high-frequency permittivity in the barrier.

Form factors have a key role to play in the e-hy scattering rate (1). For the symmetric mode they are defined as

$$
\begin{aligned}
& F_{i m(\mathrm{a})}^{\mathrm{e}-\mathrm{hy}}(Q, q)=\int_{-w / 2}^{w / 2} \mathrm{~d} z \chi_{m}(z)\left\{\sin (Q z) \chi_{i}(z)-\frac{2 \sin (Q w / 2)}{[\tanh (q w / 2)+b] \cosh (q w / 2)}\right. \\
& \left.\quad \times\left[\sinh (q z) \chi_{i}(z)-\frac{\cosh (q z)}{q} \frac{\partial}{\partial z} \chi_{i}(z)\right]\right\}
\end{aligned}
$$

and for the antisymmetric mode as

$$
\begin{aligned}
& F_{i m(\mathbf{s})}^{\mathrm{e}-\mathrm{hy}}(Q, q)=\int_{-w / 2}^{w / 2} \mathrm{~d} z \chi_{m}(z)\left\{\cos (Q z) \chi_{i}(z)-\frac{2 \cos (Q w / 2)}{[\operatorname{coth}(q w / 2)+b] \sinh (q w / 2)}\right. \\
& \left.\quad \times\left[\cosh (q z) \chi_{i}(z)-\frac{\sinh (q z)}{q} \frac{\partial}{\partial z} \chi_{i}(z)\right]\right\}
\end{aligned}
$$

where $\chi_{i(m)}$ in Eqs. (4) and (5) is the electron wave function in the subband $i(m)$. Discrete wave vector $Q$ is calculated from the boundary conditions [6].

The e-hy+pop capture rate is an average composed from the e-pop capture rate calculated according to Ref. [7] and from the e-hy capture rate (1) as

$$
\tau_{\mathrm{e}-\mathrm{hy}+\text { pop }}^{-1}=\frac{\sum_{i, m, k} f_{i}(k)\left[\lambda_{i m}^{\mathrm{e}-\mathrm{hy}}(k)+\lambda_{i m}^{\mathrm{e}-\mathrm{pop}}(k)\right]}{\sum_{i, k} f_{i}(k)},
$$

where the summation over $i$ includes only the subbands above the barrier, the summation over $m$ only below the barrier, and $f_{i}(k)$ is the electron distribution in the subband $i[1]$.

\section{Electron capture time}

Figure 1 shows the electron capture times versus the QW width obtained using the e-hy+pop and e-pop interactions in the calculation. It can be seen that the e-hy+pop interaction-induced capture time is about twice shorter than the e-pop capture time with or without screening. Its oscillations are less pronounced and the resonance minima occur at the same $\mathrm{QW}$ widths. The e-hy+pop capture time does not drop suddenly at the QW width equal to $44 \AA$ when the second bound state merges into the QW. The electrons in the low states above the barrier cannot be scattered into the second bound state in the $\mathrm{QW}$ because they are below the threshold energy for the bulk pop emission. Since the electron transition to the highest state in the QW is the dominant transition, the total e-hy+pop capture rate decreases. The same situation reappears when the third bound state merges into the QW at $w=88 \AA$. 


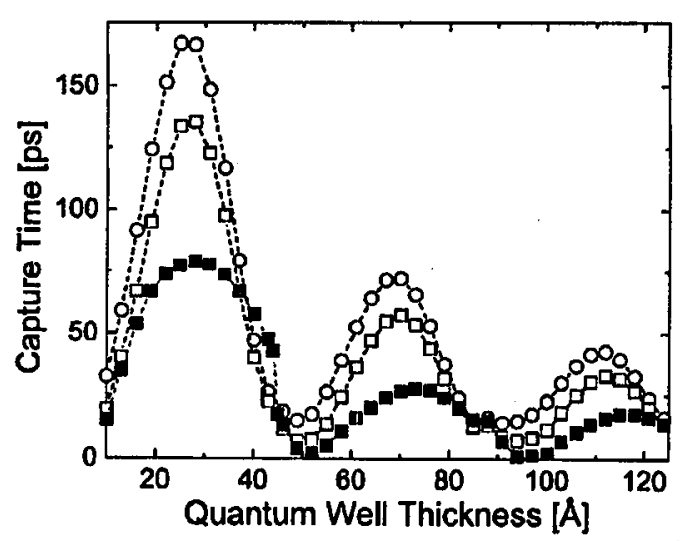

Fig. 1. e-hy +pop capture time versus $Q W$ width (full squares) as compared with the e-pop capture time when an electron interacts with the bulk phonon only. Open circles represent the statically screened e-pop interaction while open squares are for the unscreened e-pop interaction.

\section{Conclusion}

The electron capture time via the interaction of an electron with the pop has been calculated in the SCIIQW considering the confinement of phonons in the QW. We have taken an advantage of the e-hy interaction proposed by Ridley [6] which describes an electron interacting with several pop modes in a semiconductor QW. This is a more realistic description of the electron-phonon interaction and results in a shorter electron capture time which can be further improved via the e-e interaction in the resonance minima $[2,3]$.

\section{Acknowledgment}

The author would like to thank M. Moško for valuable discussions on the topic. The work has been supported by the Slovak Grant Agency for Science under contract No. 2/4057/97.

\section{References}

[1] J.A. Brum, G. Bastard, Phys. Rev. B 33, 1420 (1986).

[2] K. Kálna, M. Moško, F.M. Peeters, Appl. Phys. Lelt. 68, 117 (1996).

[3] K. Kálna, M. Moško, Phys. Rev. B 54, 13730 (1996).

[4] P.W.M. Blom, C. Smit, J.E.M. Haverkort, J.H. Wolter, Phys. Rev. B 47, 2072 (1993).

[5] B.K. Ridley, Phys. Rev. B 50, 1717 (1994).

[6] B.K. Ridley, Phys. Rev. B 47, 4592 (1993).

[7] S.M. Goodnick, P. Lugli, Phys. Rev. B 37, 2578 (1988). 